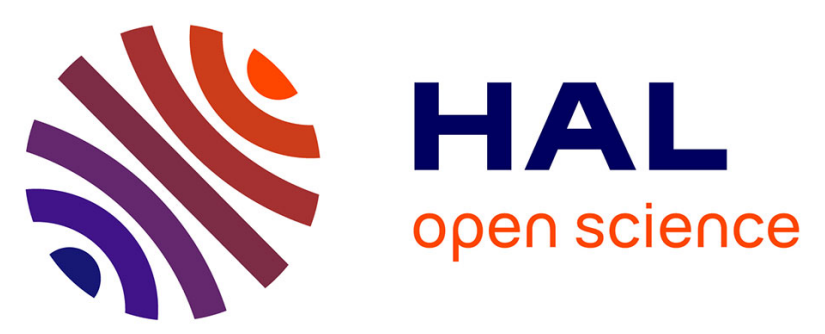

\title{
Etude du comportement dynamique des matériaux: prise en compte de l'élastoplasticité et de l'endommagement
}

\author{
N. Moulin, J. Bontaz, E. Martinez, J. Perraud
}

\section{To cite this version:}

N. Moulin, J. Bontaz, E. Martinez, J. Perraud. Etude du comportement dynamique des matériaux: prise en compte de l'élastoplasticité et de l'endommagement. Journal de Physique IV Proceedings, 1994, 04 (C8), pp.C8-571-C8-576. 10.1051/jp4:1994889 • jpa-00253450

\section{HAL Id: jpa-00253450 https://hal.science/jpa-00253450}

Submitted on 1 Jan 1994

HAL is a multi-disciplinary open access archive for the deposit and dissemination of scientific research documents, whether they are published or not. The documents may come from teaching and research institutions in France or abroad, or from public or private research centers.
L'archive ouverte pluridisciplinaire $\mathbf{H A L}$, est destinée au dépôt et à la diffusion de documents scientifiques de niveau recherche, publiés ou non, émanant des établissements d'enseignement et de recherche français ou étrangers, des laboratoires publics ou privés. 


\title{
Etude du comportement dynamique des matériaux : prise en compte de l'élastoplasticité et de l'endommagement
}

\author{
N. Moulin, J. Bontaz, E. Martinez et J. Perraud \\ CEA, CEV-M, BP. 7, 77181 Courtry, France
}

Résumé : Dans le cadre des études qui concernent la réponse des matériaux soumis à de fortes sollicitations dynamiques, la prise en compte de leur comportement élastoplastique s'est avérée nécessaire pour améliorer les restitutions numériques d'expériences, au regard d'un traitement hydrodynamique pur. Cependant, il apparaît que la prise en compte de l'endommagement du matériau améliore encore les simulations par rapport à l'expérience. Pour étudier le comportement des matériaux dans ce domaine, nous développons l'exploitation des chocs de plaques à partir des méthodes d'analyse lagrangienne.

\begin{abstract}
Some investigations have shown that dynamic behavior of materials can't be described by assuming that the solid behavior as an ideal fluid. We must take into account the strength and the spall effects.

In second part, using a lagrangian analysis method, we show that the kinematical and thermodynamical parameters of materials under shock-loading can be obtained through particle velocity and stress measurements.
\end{abstract}

\section{1-INTRODUCTION}

Dans le cadre des études concernant la réponse des matériaux soumis à de fortes sollicitations dynamiques, la prise en compte de leur comportement élastoplastique est nécessaire. Il existe un certain nombre de modèles de comportement susceptibles de prendre en compte ce caractère particulier. Une analyse préliminaire, proposant une évaluation comparative de ces modèles par le biais de restitutions numériques d'expériences $/ 1$, ne saurait être complète, sans une étude approfondie du modèle de ZERILLI-ARMSTRONG $/ 2 \%$. Cependant, dans certaines conditions, nous devons tenir compte de l'endommagement du matériau. Ce phénomène fera l'objet de la dernière partie de l'article.

\section{2- LE MODELE DE ZERILLI-ARMSTRONG}

Le modèle de ZERILLI-ARMSTRONG, inspiré très largement de la théorie régissant le mouvement des dislocations dans un matériau, propose une expression de la contrainte d'écoulement $Y$ en fonction de la déformation plastique équivalente, de la vitesse de déformation $\varepsilon$, de la température $T$, du diamètre moyen d'un grain 1 , et de la structure cristallographique du matériau étudié.

-dans le cas d'un matériau cubique faces centrées :

$Y=C_{o}(l)+C_{2} \varepsilon^{1 / 2} \exp \left(-C_{3} T+C_{4} T \ln \varepsilon\right)$

- dans le cas d'un matériau cubique centré :

$$
Y=C_{0}(l)+C_{1} \exp \left(-C_{3} T+C_{4} T \ln \varepsilon\right)+C_{5} \varepsilon^{n}
$$

\section{3-DETERMINATION DES COEFFICIENTS ASSOCIES POUR LE TANTALE}

La formulation mathématique de la contrainte d'écoulement pour le tantale, matériau de structure cristallographique cubique centrée, apparaît soús la forme (2). Six constantes sont à déterminer : $\mathrm{Co}, \mathrm{C} 1, \mathrm{C} 3, \mathrm{C} 4, \mathrm{C} 5$ et $\mathrm{n}$. 
Afin de déterminer les coefficients associés, nous avons utilisé les résultats de compression par barres d'HOPKINSON en température. Les résultats expérimentaux sont disponibles dans un large domaine de vitesses de déformation $\left(10^{-4}<\dot{\varepsilon}<2.10^{+3} \mathrm{~s}^{-1}\right)$ et dans un intervalle de températures balayant de l'ambiante jusqu'à $600^{\circ} \mathrm{C}$.

Par régression sur ces variables, nous avons obtenu les résultats rassemblés dans le tableau 1

\begin{tabular}{|c|c|c|c|c|c|}
\hline Co (MPa) & Cl (MPa) & C3 (K-1) & C4 (K-1) & C5 (MPa) & $\mathbf{n}$ \\
\hline 150 & 973 & 0,0064 & 0,0003 & 461 & 0,505 \\
\hline
\end{tabular}

Tableau 1 : Coefficients associés au modèle de ZERILLI-ARMSTRONG pour le tantale.

\section{4-COMPARAISON DES PREDICTIONS DU MODELE AVEC LES ESSAIS D'IMPACT DE CYLINDRE.}

Une évaluation du modèle peut être faite en comparant les prédictions numériques bidimensionnelles du modèle de ZERILLI-ARMSTRONG avec des résultats d'essais de TAYLOR sur le tantale.

Un cylindre de tantale, animé d'une vitesse $V p=144 \mathrm{~m} / \mathrm{s}$, vient impacter un mur indéformable. Afin de quantifier le bon accord entre l'expérience et le calcul bidimensionnel, nous nous proposons d'étudier la géométrie finale de ce demier à l'aide d'une erreur moyenne définie comme suit, par HOLMQUIST et JOHNSON / $/$,

$$
E=\frac{1}{3}\left[\left|\frac{\Delta L}{L_{t}}\right|+\left|\frac{\Delta D}{D_{t}}\right|+\left|\frac{\Delta H}{H_{t}}\right|\right]
$$

où $\mathrm{Lt}, \mathrm{Dt}, \mathrm{Ht}$ sont respectivement la longueur expérimentale du cylindre au temps $t$, Dt son diamètre au temps $t$ et $\mathrm{Ht}$ son diamètre à l'abscisse 0.2 au temps $t$.

Si L, D, $H$ définissent les mêmes dimensions pour les simulations numériques, on a :

$$
|\Delta \mathbf{L}|=\left|\mathbf{L}_{\mathrm{t}}-\mathbf{L}\right|(4) ;|\Delta \mathrm{D}|=\left|\mathrm{D}_{\mathrm{t}}-\mathrm{D}\right|(5) ;|\Delta \mathrm{H}|=\left|\mathrm{H}_{\mathrm{t}}-\mathrm{H}\right|(6)
$$

Afin de mettre en évidence les bons résultats du modèle de ZERILLI-ARMSTRONG, nous avons comparé ces derniers à ceux obtenus à l'aide des modèles de STELNBERG-COCHRAN-GUINAN /4/ (figure 1) et de JOHNSON-COOK $/ 5 /$.

L'ensemble des résultats est résumé dans le tableau 2.[Lf: longueur finale de l'échantillon récupéré

\begin{tabular}{|c|c|c|c|c|}
\hline Modèles EP & SCG & J. COOK & Z.A. & Expérience \\
\hline $\mathrm{Lf}(\mathrm{cm})$ & 1,330 & 1,245 & 1,270 & 1,308 \\
\hline $\mathbf{R f}(\mathrm{cm})$ & 0,580 & 0,630 & 0,614 & 0,615 \\
\hline $\mathrm{E}(\%)$ & $\mathbf{4 , 0}$ & 2,8 & 1,8 & - \\
\hline
\end{tabular}

Tableau 2 : Résultats sur le tantale, obtenus à température ambiante pour trois modèles élastoplastiques.
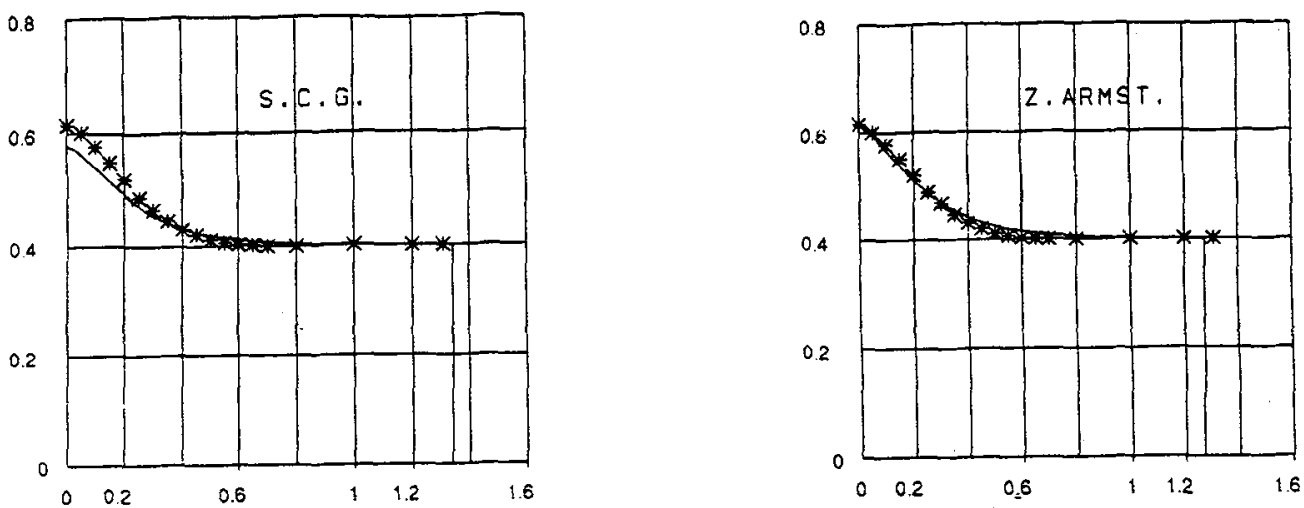

Figure 1: Etude de la géométrie finale de l'échantillon de tantale.(*) Profil expérimental 


\section{5-CONFRONTATION DES PREDICTIONS DES MODELES AVEC DES ESSAIS DE CHOCS DE PLAQUES}

Afin d'évaluer le modèle de ZERLLI-ARMSTRONG dans des domaines encore plus fortement dynamiques que celui de l'essai de TAYLOR, nous utilisons des expériences de chocs de plaques avec une mesure de vitesse matérielle derrière une fenêtre de fluorure de lithium $/ 6 /$.

Un lanceur à poudre de $60 \mathrm{~mm}$ de diamètre permet au projectile d'atteindre des vitesses variant de 100 à $1600 \mathrm{~m} / \mathrm{s}$; l'impacteur conduit par un sabot de polyéthylène va engendrer dans l'échantillon (cible), une onde de choc.

Selon les impédances de choc des matériaux constituant le projectile, le matériau étudié subira un choc suivi d'une détente (choc-détente), un choc suivi d'un second choc (choc-rechoc) ou des sollicitations plus complexes telles que le choc-detente-rechoc.

Les mesures de la vitesse matérielle à l'interface cible/LiF sont réalisées par la technique d'interférométrie doppler laser (I.D.L.), à travers une fenêtre de fluonure de lithium $/ 6 /$.

Nous allons illustrer nos propos à l'aide d'un essai choc-détente-rechoc, réalisé sur du tantale, caractérisé par le tableau 3.

La vitesse du projectile mesurée par I.D.L. est $\mathrm{Vp}=700 \pm 6 \mathrm{~m} / \mathrm{s}$

\begin{tabular}{|c|c|c|c|c|c|c|}
\cline { 2 - 6 } \multicolumn{1}{c|}{} & \multicolumn{3}{c|}{ Impacteur } & \multicolumn{2}{c|}{ Cible } \\
\cline { 2 - 7 } & Polyéthylène & Ta & Al & Ta & Ta & LiF \\
\hline $\begin{array}{c}\text { épaisseur } \\
(\mathrm{mm})\end{array}$ & 40 & 2,04 & 2,0 & 2,04 & 3,0 & 25,0 \\
\hline
\end{tabular}

Tableau 3 : caractéristiques expérimentales du tir choc-détente-rechoc

L'évolution expérimentale de la vitesse matérielle en fonction du temps est représentée sur la figure 2. La figure 4 fait apparaître une bonne corrélation expérience /calcul 2D ZERILLI-ARMSTRONG.

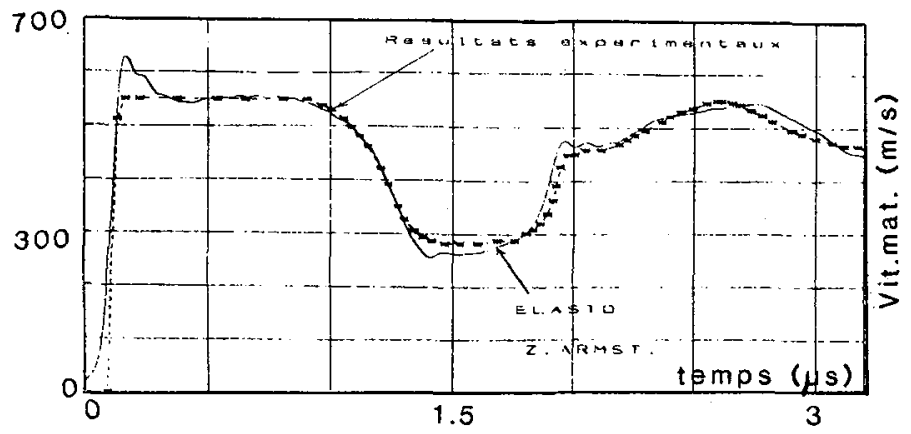

Figure 2: Choc-détente-rechoc sur tantale. Confrontation expérience /calcul ZA (2D)

Cependant, dans ces domaines très fortement dynamiques, il est nécessaire d'accéder directement aux évolutions temporelles des principaux paramètres : la contrainte $\sigma$, la déformation $\varepsilon$, la vitesse de déformation $\dot{\varepsilon}$, etc. ... . Pour cela, nous développons l'exploitation d'un impact plan à partir des méthodes d'analyse lagrangienne $/ 7 /, / 8 /, / 9 /, / 10 /, / 11 /$. 


\section{6-EXPLOITATION DES PROFILS EXPERIMENTAUX A PARTIR DES METHODES D'ANALYSE LAGRANGIENNE.}

Dans un milieu matériel, la propagation d'une onde de choc entraîne une modification des caractéristiques mécaniques et thermodynamiques de l'échantillon. L'exploitation des profils, de contrainte ou de vitesse matérielle mesurés à différents niveaux dans le matériau, permet, à partir des méthodes d'analyse lagrangienne, l'élaboration et la mise au point de modèles de comportement.

En effet, sans aucune hypothèse sur la loi de comportement ou l'équation d'état du matériau étudié, ces méthodes permettent de décrire l'évolution temporelle de divers paramètres tels que, la contrainte, le volume ou l'énergie interne spécifique.

Les relations mises en oeuvre sont établies à partir des lois fondamentales de la mécanique des milieux continus; en coordonnées de lagrange et dans le cas du mouvement monodimensionnel plan, ces dernières s'écrivent:

- Conservation de la masse :

$$
\begin{aligned}
& \frac{1}{\rho_{o}}\left(\frac{\partial u}{\partial h}\right)_{t}-\left(\frac{\partial V}{\partial t}\right)_{h}=0 \\
& \frac{1}{\rho_{o}}\left(\frac{\partial \sigma}{\partial h}\right)_{t}+\left(\frac{\partial u}{\partial t}\right)_{h}=0 \\
& \frac{1}{\rho_{o}}\left(\frac{\partial u}{\partial h}\right)_{t}+\left(\frac{\partial e}{\partial t}\right)_{t}=0
\end{aligned}
$$

avec $\mathbf{u}$ : vitesse matérielle

$\mathrm{V}$ : volume spécifique

$\sigma:$ contrainte dans la direction du mouvement

$\rho_{0}$ :masse volumique initiale

e : énergie interne spécifique

$\mathrm{h}$ : coordonnée de lagrange

Sous certaines hypothèses telles que : un choc monodimensionnel plan et un processus adiabatique, lintégration de (7), (8) et (9) à h constant sur un court intervalle de temps (t-to) conduit à :

$$
\begin{aligned}
& u(h, t)=u\left(h, t_{o}\right)-\frac{1}{\rho_{o}} \int_{t_{o}}^{t}\left(\frac{\partial \sigma}{\partial h}\right)_{t} d t \\
& V(h, t)=V\left(h, t_{o}\right)+\frac{1}{\rho_{o}} \int_{t_{o}}^{t}\left(\frac{\partial u}{\partial h}\right) d t \\
& e(h, t)=e\left(h, t_{o}\right)-\frac{1}{\rho_{o}} \int_{t_{o}}^{t} \sigma\left(\frac{\partial u}{\partial h}\right) d t
\end{aligned}
$$

Ainsi, la mesure de $\sigma(t)$ permet d'accéder à $u(t), V(t)$ et $e(t)$; de même la mesure de $u(t)$ permet de calculer $\sigma(t), V(t)$ et $e(t)$.

Il apparaît donc que l'exploitation des profils de contrainte et/ou de vitesse matérielle permet de déterminer l'évolution des grandeurs cinétiques et thermodynamiques du matériau sous choc.

Soit le test d'impact plan décrit précédemment (figure 2), la mesure des profils expérimentaux de contrainte et de vitesse matérielle est effectuée aux positions:

$$
\begin{aligned}
& \mathrm{h} 1=10 \mathrm{~mm}, \\
& \mathrm{~h} 2=12 \mathrm{~mm}, \\
& \mathrm{~h} 3=16 \mathrm{~mm}, \text { par rapport à la surface d'impact. } \\
& \text { avec e impacteur }=4 \mathrm{~mm},
\end{aligned}
$$

L'exploitation numérique de ces signaux (contrainte ou vitesse matérielle) permet d'accéder aux évolutions temporelles :

- de la déformation longitudinale

- du volume spécifique, et à des profils tels que la contrainte en fonction de la déformation (fig.3) 


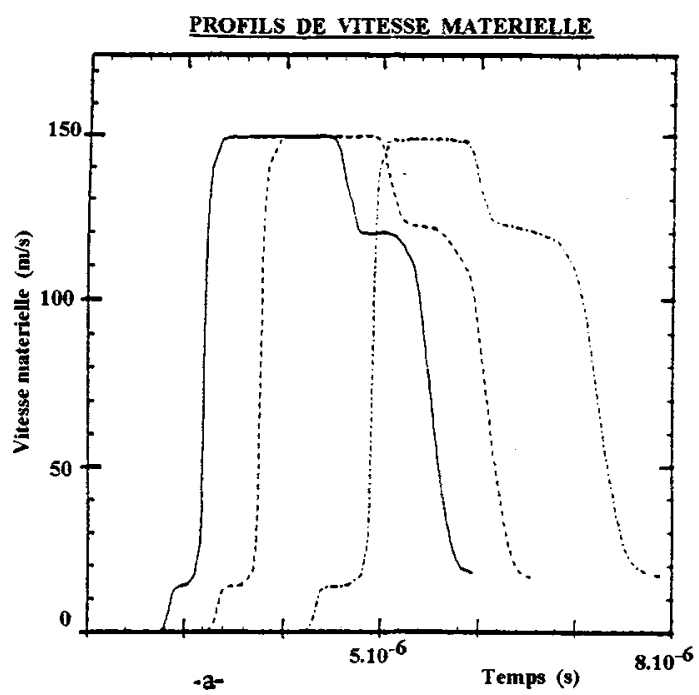

Figure 3 : Impact plan d'une cible en tantale
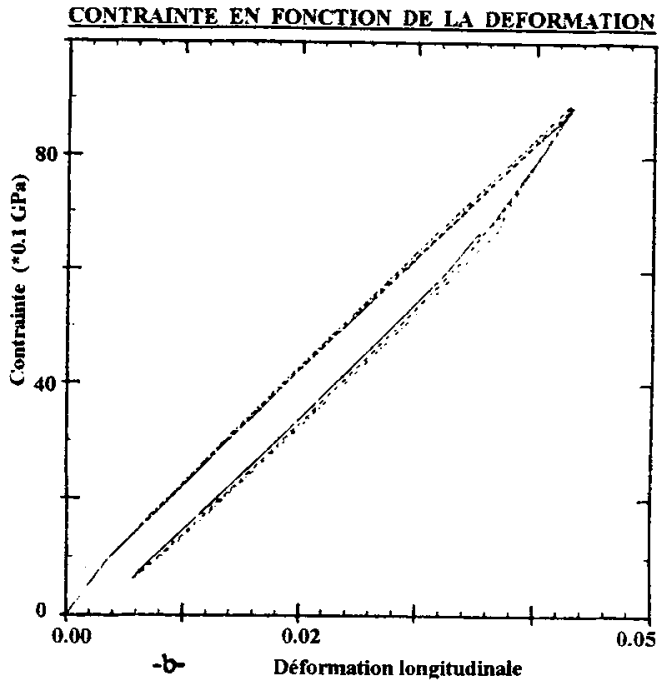

b) Contrainte en fonction de la déformation

Ainsi, en vue de l'élaboration et la mise au point des modèles de comportement, les méthodes d'analyse lagrangienne s'avèrent être particulièrement intéressantes.

\section{7- ENDOMMAGEMENT - RUPTURE.}

Dans certaines configurations expérimentales de chocs de plaques (figure 2), il est nécessaire d'associer à l'étude du comportement élastoplastique celle de lendommagement - rupture. La figure 6 montre la comparaison entre la simulation sans endommagement et l'expérience.

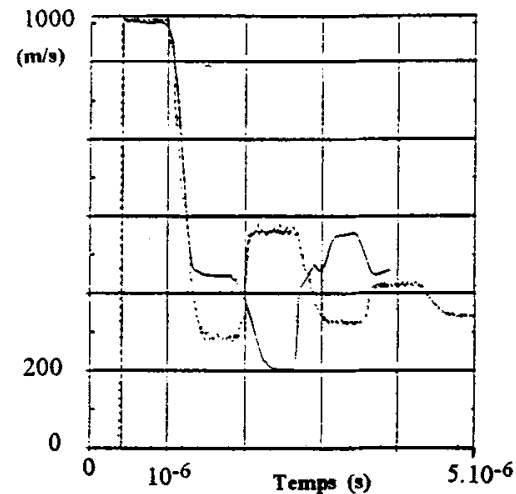

Figure 5:

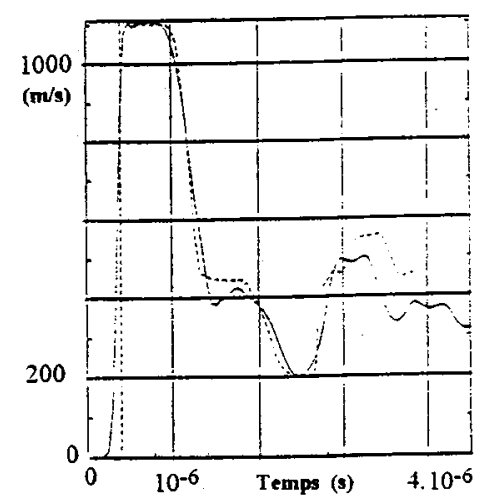

Figure 6

Vitesse de surface du cuivre en fonction du temps. Vitesse de surface du cuivre en fonction du temps.

Vitesse d'impact $=1600 \mathrm{~m} / \mathrm{s}$

Projectile $=2 \mathrm{~mm}$ (cuivre) . Cible $=2 \mathrm{~mm}$ (cuivre)

- : Expérience

$\cdots$ :-Simulation sans critère d'endommagement Comparaison expérience / simulation 2D avec le critère Tuler-Butcher.

--- :Expérience

_.:Simulation avec critère d'endommagement.

L'introduction d'un critère simple de tension d'écaillage, permet d'améliorer la restitution numérique de l'expérience. A partir, d'un calcul monodimensionnel avec prise en compte d'un critère tension 
d'écaillage, la position de l'écaille apparaît à environ $0.3 \mathrm{~mm}$ de l'interface projectile-cible et entre 0.7 et 0.85 microsecondes; position correspondant à la rencontre des ondes de détentes.

Une démarche identique a été effectuée avec un calcul bidimensionnel et un critère Tuler Butcher /12/,/13/: avec $\lambda=2, \mathrm{~K}_{\mathrm{crit}}=0.7510^{9}\left(\mathrm{~Pa}^{2} 10^{-6} \mathrm{~s}\right)$ et $\sigma_{\mathrm{S}}$ contrainte seuil $=0.510^{9} \mathrm{~Pa}$ au temps ts.

$$
\int_{\mathrm{ts}}^{\mathrm{t}}\left(\operatorname{Max}\left(\sigma_{\mathrm{I}}, \sigma_{\Pi}, \sigma_{\mathrm{III}}\right)-\sigma_{\mathrm{S}}\right)^{\lambda} \mathrm{dt}>\mathrm{K}_{\mathrm{crit}}
$$

La simulation met en évidence un début d'endommagement (tension $>0.5 \quad 10^{9} \mathrm{~Pa}$ ) à $0.87 \quad 10^{-6}$ secondes et une fracture dans le projectile à $0.9610^{-6}$ secondes qui apparaît entre $0.2 \mathrm{~mm}$ et $0.4 \mathrm{~mm}$ de l'interface: projectile-cible.

La comparaison expérience-calcul 2D avec le critère Tuler Butcher est représenté figure 5.

La bonne corrélation entre les valeurs expérimentales et la nouvelle simulation permet de croire à une meilleure interprétation du phenomène avec ce nouveau critère.

\section{CONCLUSION.}

Nous savons qu'un traitement hydrodynamique est insuffisant pour restituer nos expériences fortement dynamique. La prise en compte du comportement élastoplastique par l'intermédiaire du modèle Zerilli-Armstrong ainsi que l'endommagement du matériau à l'aide de critères de rupture tel que TulerButcher est nécessaire. Nous disposons, en outre, d'un outil d'exploitation des profils expérimentaux l'analyse lagrangienne qui nous permet d'accéder à l'évolution temporelle de certaines grandeurs caractéristiques du matériau.

\section{/1/. N. MOULIN}

\section{REFERENCES}

Influence de l'élastoplasticité sur le comportement dynamique des matériaux. Approches expérimentale et numérique. Dymat, 7 ème journée d'études, I.S.L., 7-8/10/1992.

/2/. F.J ZERILLI, R.W.ARMSTRONG

Dislocation mechanics based constitutive relations for material dynamics calculations. J. Appl.Phys 61 (5), 1987.

/3/. T.J.HOLMQUIST, G.R. JOHNSON

Determination of constants and comparaison of results for versions constitutive models

J.de Phys.IV, coll C3, 1991-supp du J. de phys III, vol 1

14/. D.J STEINBERG, S.G COCHRAN , M.W. GUINAN

A constitutive model for metals applicable at high strain rate. J. Appl.Phys., 51, 1498,1980

15/. G.R.JOHNSON,W.H. COOK

A constitutive model and data for metals subjected to large strains, high strain rates and high temperature.

proc. $7^{\text {th }}$ Int.Symp. on Ballistic, pp. 541-547, The Hague, 1983

16\%. R.COURCHINOUX, P.CHAPRON, P. ELIAS

Particle velocity profiles mesured by LDI through a LiF windows in loading unloading experiments.

Shock compression of condensed matter, pp 771-774, 1991.

/7/. G.R. FOWLES, R.F.WILLIAMS

Plane stress wave propagation in solids.J. of Appl. Physics, vol.41 (1) (January 70)

/8/. M. COWPERTHWAITE , R.F.WILLIAMS

Determination of constitutive relationships with multiple gauges in nondivergent waves.J. Appli.Physics, vol.42 (January 71 )

19/. L.SEAMAN

Lagrangian analysis for multiple stress or velocity gauges in attenuating waves.J. of Appl. Physics, vol.45 (10) (october 74)

/10/. J.CAGNOUX, P.CHARTAGNAC, P.HEREIL and M.PEREZ

Lagrangian analysis. Modern tool of the dynamics of solids-Ann. Phys. (Paris) (12), 451 (1987)

/11/. J.B. ADDUN, Y.M.GUPTA

Analysis of lagrangian gauge measurements of simple and nonsimple plane waves.J.of Appl. Physics, vol.69 (10) (May 199I)

/12/. F.R.TULER, B.M.BUTCHER

A criterion for the time dependence of dynamic fracture. Int. J.Fract. Mech. 4 (1968) 431

113/. J.BLESS.

Spall criteria for several metals, University of Dayton Research Institute. Final report 02/1979-12/1980. 